\title{
Beta relaxation in the shear mechanics of viscous liquids: Phenomenology and network modeling of the alpha-beta merging region
}

\author{
Bo Jakobsen *, Kristine Niss, Claudio Maggi, Niels Boye Olsen, Tage Christensen, Jeppe C. Dyre \\ DNRF Centre “Glass and Time", IMFUFA, Department of Sciences, Roskilde University, Postbox 260, DK-4000 Roskilde, Denmark
}

\section{A R T I C L E I N F O}

\section{Article history:}

Received 15 April 2010

Received in revised form 2 August 2010

Available online 21 September 2010

\section{Keywords:}

Viscous liquids;

Shear modulus:

Glass-forming liquids;

Beta relaxation;

Mechanical modelling

\begin{abstract}
A B S T R A C T
The phenomenology of the beta relaxation process in the shear-mechanical response of glass-forming liquids is summarized and compared to that of the dielectric beta process. Furthermore, we discuss how to model the observations by means of standard viscoelastic modeling elements. Necessary physical requirements to such a model are outlined, and it is argued that physically relevant models must be additive in the shear compliance of the alpha and beta parts. A model based on these considerations is proposed and fitted to data for Polyisobutylene 680 .
\end{abstract}

(c) 2010 Elsevier B.V. All rights reserved.

\section{Introduction}

Most knowledge about the beta (secondary) relaxation process in glass-forming liquids has been obtained from dielectric spectroscopy. This is due to the abundant amount of data available from this technique, its high sensitivity and wide dynamical range (routinely covering more than 9 decades). Dielectric spectroscopy, however, does not probe the mechanical relaxation processes in the liquid. It is therefore important to know to what extent the dielectric phenomenology carries over to the mechanical relaxation functions. An example where phenomenology does not carry over is the case of the large Debye-like peak observed in the dielectric loss of monoalcohols, which does not have any observable signature in the frequency-dependent shear modulus [1]. There are also cases where dielectric spectroscopy is not well suited for monitoring the liquid's relaxations, e.g., if the liquid molecules have a very small dipole moment (e.g., Squalane [2]) or if the spectrum is dominated by other effects (e.g., dc conduction).

This motivates the interest in exploring the phenomenology of the beta relaxation in other response functions. In the Roskilde group we have developed a technique [3] for measuring the frequencydependent complex shear modulus, $G(\omega)=G^{\prime}(\omega)+i G^{\prime \prime}(\omega)$, which covers a rather large frequency range compared to other mechanical spectroscopy techniques, $1 \mathrm{mHz}-10 \mathrm{kHz}$, over moduli ranging from $0.1 \mathrm{MPa}$ to $10 \mathrm{GPa}$. The technique is based on piezoelectric ceramics acting as converter from mechanical to electrical impedance. Our setup consists of a piezoelectric shear-modulus gauge (PSG) in

\footnotetext{
* Corresponding author.

E-mail address: boj@ruc.dk (B. Jakobsen).
}

conjuntion with a cryostat setup [4] and an electronics setup for measuring the frequency-dependent capacitance [5].

With this technique the dynamical range is large enough to investigate the beta relaxation in the shear mechanics. Fig. 1 shows examples of shear-mechanical spectra in two cases where the beta relaxation is very well resolved due to its position at low frequency. We have in the past obtained shear-mechanical data on several liquids, with and without a clearly resolvable beta relaxation ${ }^{1}$; in most cases dielectric data have also been taken (or exist in the literature). These measurements constitute a database large enough for inquiring into the general properties of the beta relaxation in shear mechanics and the relation to the corresponding process in the dielectric response.

In section 2 the general phenomenology of the shear-mechanical beta relaxation is presented. Besides establishing the phenomenology of the shear-mechanical beta relaxation, such data can also be used to investigate different models. An important issue is the way in which the alpha and beta relaxations merge and interact in the region where

\footnotetext{
1 Data have been taken on the following substances (see the original publications for details): 1,3-Butanediol [6], 1,2,6-Hexanetriol [7], Tetramethyltetraphenyltrisiloxane (DC704) [6,8,9], 2-Methyl-2,4-pentanediol [3], Dibutylphthalate (DBP) [9-11], Triphenylethylene (TPE) [8,9], Polyphenylether (PPE) [8,9], Perhydrosqualene (Squalane) [8,9], Polybuta- diene [8,9], Decahydroisoquinoline (DHIQ) [8,9], Tripropyleneglycol (TPG) [8,9], Poly- isobutylene (PIB680) [12] (this work [9]), Pentaphenyltrimethyltrisiloxane (DC705) [9,11], 1,2-Propanediol [9,11], Diethylphthalate (DEP) [9,11], m-Toluidine [9,11], 2Butanol [1,9], 2-Ethyl-1-hexanol [1,9]. Most of the data are available in electronic form from the "Glass and Time: Data repository" [9], where figures of the available datasets can also be found. The Polyisobutylene (PIB680) data is on a sample with molecular weight $M_{w}=680 \mathrm{~g} / \mathrm{mol}$ and polydispersity $M_{w} / M_{n}=1.06$. The sample was acquired from Polymer Standard service, and used as received. Based on the shear mechanical data the glass transition temperature was found to be $T_{g}=195 \mathrm{~K}$, and the fragility index $m=80$ [12].
} 


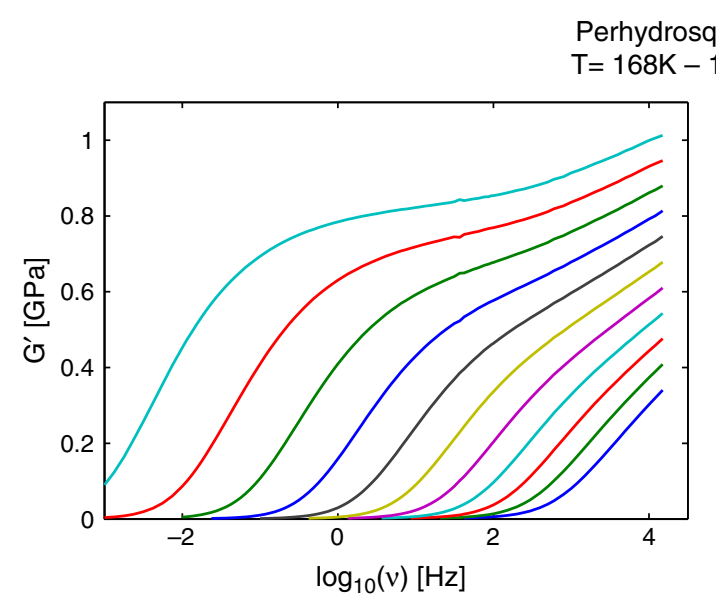

Perhydrosqualene (Squalane)

$\mathrm{T}=168 \mathrm{~K}-188 \mathrm{~K}$ in steps of $2 \mathrm{~K}$

Polyisobutylene (PIB680)

$\mathrm{T}=198 \mathrm{~K}-234 \mathrm{~K}$ in steps of $2 \mathrm{~K}$
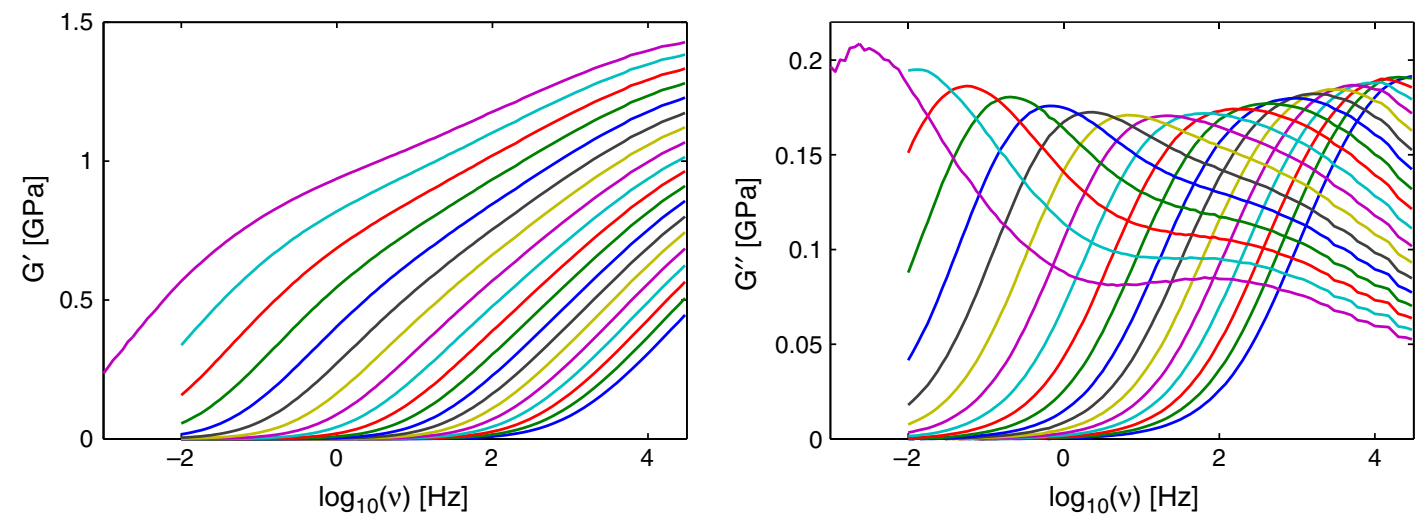

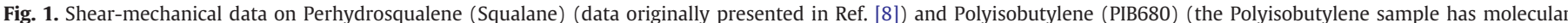

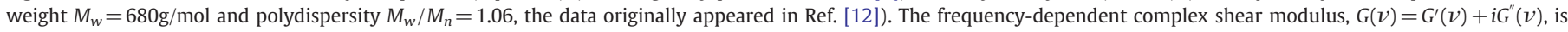
presented for each liquid for a number of isothermal frequency scans, showing real and imaginary parts.

their time scales are not well separated. Because the shear modulus probes a fundamental property of the liquid, namely its flow properties, constraints can be put on which models are physically acceptable. In section 3 we present such considerations based on the class of simple element-based models. In section 4 a specific model is presented, which is able to capture the full spectral shape during merging of the alpha and beta processes, as shown by the example of Polyisobutylene. Section 5 summarizes the conclusions.

\section{Phenomenology of the shear-mechanical beta relaxation: Comparing to the dielectric beta relaxation}

The below phenomenology of shear-mechanical beta relaxations in molecular liquids is based on a limited number of data sets, because few techniques exist that can access the relevant frequency and modulus ranges. Besides the above-mentioned measurements by our group, we know of the following works comparing to the dielectric phenomenology: The work on m-Toluidine by Mandanici et al. [13] (see also Ref. [14]) and the work on Ethylcyclohexane by Mandanici and Cutroni [15,16] (see Ref. [17] for the dielectric data).

The below three points were in 2005 proposed by some of us based on measurements on seven liquids [8], and are largely substantiated by the data sets existing today. The entire shear-mechanical beta relaxation peak is rarely visible within our experimental frequency window, a fact that obviously complicates the analysis quite a lot. We interpret the high-frequency rise, which is sometimes seen in the imaginary part of the shear modulus (see e.g., the Squalane data in Fig. 1), as the signature of a beta relaxation process peaking at a frequency that is outside our measuring range. The observations are based on this interpretation of the behavior of the high-frequency rise in the imaginary part of the shear modulus.

1 When a beta process is observed in the shear mechanics, it is also observed in dielectrics, and vice versa.

In most cases this is a very clear observation; either a liquid has a beta process (e.g., Tripropyleneglycol in Fig. 2, Dibutylphthalate $[9,11,18]$ and Polybutadiene $[8,9])$, or it does not (e.g., Triphenylethylene and Tetramethyltetraphenyltrisiloxane (DC704) $[8,9]$ ).

2 The shear-mechanical beta relaxation is shifted to higher frequencies compared to the dielectric beta relaxation.

Interestingly, the same is true for the alpha relaxation, an experimental observation substantiated by many measurements from different groups, e.g., [19-24]. The shear-mechanical alpha relaxation is normally shifted from 0.5 to 1 decade up in frequency compared to the dielectric alpha relaxation at same temperature. $m$-Toluidine is an extreme case in this respect with a very small shift $[13,14]$.

3 The relaxation strength of the shear-mechanical beta relaxation, relative to the alpha relaxation, is larger than the corresponding ratio for the dielectric case.

An extreme case is that of Tripropyleneglycol (Fig. 2), in which the dielectric beta relaxation is barely visible on the scale of the alpha relaxation, whereas the shear-mechanical beta relaxation is quite pronounced.

These three points supplement the picture of the dielectric beta process detailed in three earlier publications of ours [25-27]: The dielectric beta process is well known to have a thermally activated 
Tripropyleneglycol(TPG)
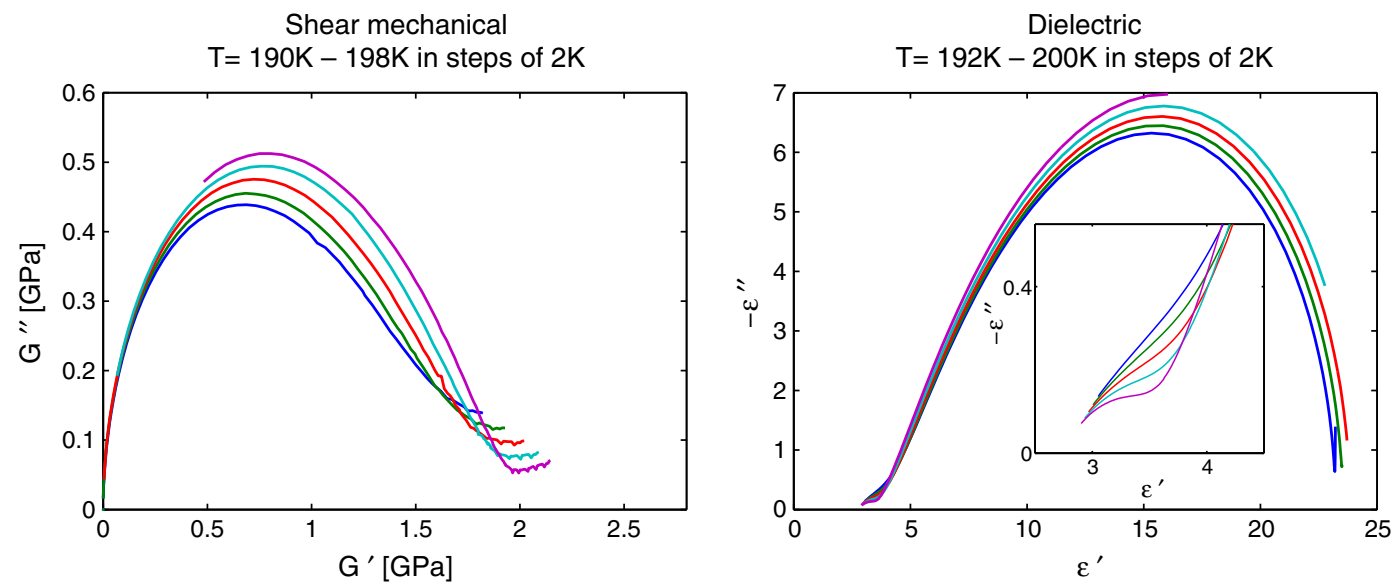

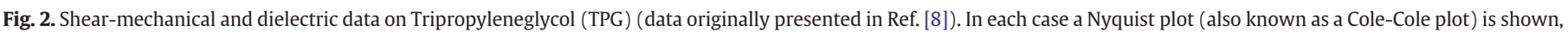
plotting the imaginary part against the real part. The inset shows a zoom-in of the dielectric beta relaxation.

loss-peak frequency in the glass phase with an almost temperatureindependent loss magnitude. In the equilibrium liquid phase, however (where it is difficult to study the dielectric beta process due to the merging with the alpha process) the characteristics of the beta process are quite different [25-27]. Here we find that the dielectric loss peak frequency is either completely temperature independent or only very weakly temperature dependent. In contrast, the beta loss peak magnitude is strongly temperature dependent in the liquid phase. In order to model these striking features, as well as the annealing behavior of the beta process, in 2003 some of us proposed a "minimal" model [27] for the beta process based on an asymmetric potential with two minima. The two energy parameters of this model were assumed to depend on one single structural parameter, $s$, which freezes in the glass phase. This model is able to account for the difference between liquid and glass behavior and the beta process annealing properties.

In the mechanical case we are unfortunately not able to study the beta process in the glass phase with our PSG transducer, because the calibration of the PSG parameters becomes uncertain at temperatures deep below the glass transition of the investigated liquid. This is due to the difference in the static stress between the filled and empty transducer. It would certainly be worthwhile to compare the mechanical beta relaxation in glass and liquid to see whether the picture is similar to what is seen for dielectrics.

Currently no solid explanation exists for the differences between the phenomenology of shear mechanical and dielectric alpha and beta relaxations. The good news is that the shear-mechanical data confirm that what we observe in dielectric spectroscopy is closely related to the basic mechanical relaxations of the liquid, also on the level of the beta process. The general shift between the time scales of the two processes, which is in most cases virtually temperature independent, is not surprising, given that such a shift often arises going from one response function to another. A striking fact is the generally observed enhancement of the relative relaxation strength of the mechanical beta processes compared to that of the dielectrics. This shows that conclusions based on dielectric spectroscopy may lead to an underestimation of the importance of the beta relaxation in the total relaxation picture.

It is often argued that a connection between dielectric and shearmechanical relaxations must exist, with ideas dating back to Debye's model of a rotating sphere, the rotation rate of which is inversely proportional to the shear viscosity [28]. It is not the purpose of this paper to review or discuss such models in detail, but it should be mentioned that some of us earlier argued $[6,29]$ that the Gemant-
DiMarzio-Bishop model [30,31] captures the qualitative differences between shear-mechanical and dielectric alpha and beta relaxations. The model is a generalization of the Debye model [28] to the case of a frequency-dependent viscosity. In Ref. [29] we argued that the correct quantities to compare are the shear modulus $G(\omega)$ and the rotational dielectric modulus, $\frac{1}{\epsilon(\omega)-n^{2}}$, where $n^{2}$ contains the contribution to the dielectric constant from electronic polarization $\left(n^{2}=1+\chi_{e}\right.$, with $\chi_{e}$ characterizing the electronic polarizability). This model is qualitatively consistent with points 1-3 above. The model, however, does not fit data quantitatively (generally, it is not possible to get the correct shape and position of the alpha peak of the dielectric rotational modulus compared to the shear modulus (see also Ref. [6]); the model furthermore predicts too large a beta relaxation in the shear modulus).

Phenomenologically it is sometimes suggested that what should be compared is the "dielectric modulus", $1 / \epsilon(\omega)$, and the shear modulus. However, in our opinion this is unphysical as one can write $\epsilon(\omega)=\chi_{r}(\omega)+n^{2}$, where $\chi_{r}(\omega)$ is the contribution from rotation of the molecules. $\chi_{r}(\omega)$ is the quantity that is assumed to be related to the shear modulus/viscosity. The consequence is that $\frac{1}{\epsilon(\boldsymbol{\omega})}=\frac{1}{\chi_{\mathrm{r}}(\boldsymbol{\omega})+n^{2}}$, hence that two liquids with the same $\chi_{r}(\omega)$ (and therefore same molecular rotational dynamics) can have different dielectric modulus; a related discussion took place in the 1990's in the ion conduction field, see, e.g., [32-34]. The quantity arising naturally from the Gemant-DiMarzio-Bishop model [29], the rotational dielectric modulus, $\frac{1}{\epsilon(\omega)-n^{2}}=\frac{1}{\chi_{r}}$, is on the contrary only related to the rotational part of the dielectric constant.

\section{Considerations on how to fit the full shear-mechanical spectrum}

There are several ways to model the phenomenology of the interplay between the alpha and beta relaxation processes. A recent paper by some of us [35] presents a new approach for the dielectric alpha-beta merging and also briefly reviews other approaches. Below paper we present a related scheme for the alpha-beta merging in shear-mechanical data.

When formulating models for the alpha-beta relaxation, the interplay of a number of physical constraints must be taken into account. These constraints limit the number of possible models for the full shear-mechanical spectrum, while there are no similar constraints for modeling the dielectric spectrum. 
The following analysis is limited to the classical class of macroscopic viscoelastic models as described, e.g., by Harrison [36]. The set of fundamental mechanical components consists of
1) an elastic spring :
$J(\omega)=1 / G$
2) a dash pot:
$J(\omega)=(i \omega \eta)^{-1}$
3) a constant-phase element: $J(\omega)=k(i \omega)^{-\alpha}$ with $0 \leq \alpha \leq 1$,

where $J(\omega)$ is the complex frequency-dependent compliance $(J(\omega)=1$ / $G(\omega)$ ). The elements are characterized by 1 ) a spring constant $(G), 2)$ the viscosity $(\eta)$, and 3 ) a strength $(k)$ and an exponent $(\alpha)$. The constant phase element was considered long ago by Cole and Cole in the dielectric context [37] and later by Jonscher [38-40]; it generalizes the $\sqrt{i \omega \tau}$ element of the BEL model by Barlow et al. [41]. These elements can be connected in two different ways, a mechanical series connection (with same stress on each element and additive displacement, yielding additive shear compliances) or a mechanical parallel connection (with same displacement on each element and additive stress, implying additive shear moduli). ${ }^{2}$

Combining such elements to model an observed response is today often regarded as old-fashioned providing little insight. We do see advantages with this kind of phenomenological modeling, however; for instance combining the elements will always lead to a model that is consistent with basic requirements like causality, analyticity, and positive dissipation. Possible connections between the alpha and beta relaxation (e.g. Refs. [25-27]) may in some cases be integrated into this kind of models through parametric relations between the elements. However, as the models are intrinsically coarse grained, information and correlations on the more detailed level are lost (as e.g. information on correlations on the level of dynamical heterogeneity [42]). Of course, this kind of modeling should only be regarded as a step towards the ultimate, microscopic model, which will eventually provide a complete description of the mechanical response of viscous liquids.

Physical constraints on the possible models come from the knowledge of the limiting behavior of the shear-mechanical response functions:

$\lim _{\omega \rightarrow \infty} G^{\prime}(\omega)=G_{\infty}$

$\lim _{\omega \rightarrow 0} G^{\prime}(\omega)=0$

$\lim _{\omega \rightarrow 0} \eta(\omega)=\lim _{\omega \rightarrow 0} G^{\prime \prime}(\omega) / \omega=\eta_{0}<\infty$.

The physical interpretation of these equations are as follows: (1) at short times a liquid behaves as a pure spring, (2) at long times the liquid's stresses relax to zero, (3) the dc limit of the viscosity is a finite (real) constant.

Before discussing the merging of the alpha and beta relaxation we need to choose models for the individual relaxations. We found that a generalized BEL model can fit the pure alpha relaxation very well. The generalized BEL model is illustrated in Fig. 3a. It consists of a mechanical series connection of a spring, a dash pot, and a constantphase element (hence, it can be seen as a generalization of the Maxwell model). The complex frequency-dependent modulus is given by

$G(\omega)=\frac{G_{\infty, \alpha}}{1+\frac{1}{i \omega \tau_{\alpha}}+q\left(\frac{1}{i \omega \tau_{\alpha}}\right)^{\alpha}}$,

where $\tau_{\alpha}=\frac{\eta}{G_{\infty}}$ (the Maxwell relaxation time), and $q=G_{\infty, \alpha} k_{\alpha}\left(\tau_{\alpha}\right)^{\alpha}$ in terms of the quantities defining the individual model components.

\footnotetext{
2 The mechanical networks described herein can alternatively be expressed as electrical circuits by representing mechanical displacement as charge displacement and stress as voltage. The spring is then replaced by a capacitor and the dash pot by a resistor. Parallel and series connections are furthermore interchanged.
}

a)

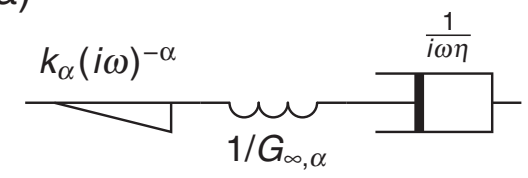

b)

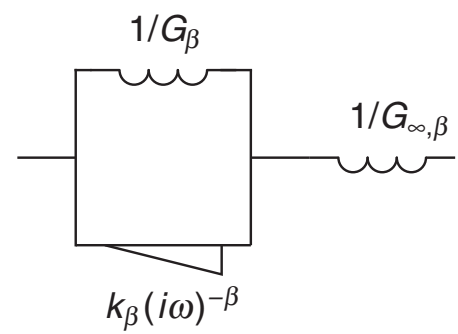

C)

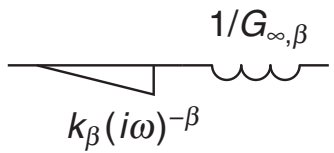

Fig. 3. Mechanical network representation of the discussed individual model components. The building blocks are springs, dash pots, and constant-phase elements. The shear compliance is indicated for the individual elements. a) Generalized BEL model used for the shear-mechanical alpha relaxation (modulus expressed in Eq. (4)). $\mathrm{b}$ and c) Cole-Cole like mechanical models used for modeling the mechanical beta relaxation (modulus expressed in Eqs. (5) and (6) respectively).

It is well known that the dielectric beta relaxation can be fitted by a Cole-Cole type function, which is symmetric in a log-log plot. From the shear-mechanical data (e.g., Fig. 1) it is observed that the shearmechanical beta relaxation is also very broad and has non-Debye lowfrequency side. Since the information on the high-frequency side of the mechanical beta relaxation is limited, we assume that the mechanical beta process is also symmetrically broadened; we thus fit it with a Cole-Cole type model.

There are two possible Cole-Cole type mechanical models giving a peak in the modulus. Figs. $3 \mathrm{~b}$ and $\mathrm{c}$ show these two models, which have shear modulus given by, respectively,

$$
\begin{aligned}
& G(\omega)=\frac{G_{\infty, \beta}}{1+\frac{G_{\infty, \beta} / G_{\beta}}{1+\left(i \omega \tau_{\beta, J}\right)^{\beta}}} \text { for model }(\mathrm{b}) \\
& G(\omega)=\frac{G_{\infty, \beta}}{1+\left(\frac{1}{i \omega \tau_{\beta}}\right)^{\beta}} \text { for model }(\mathrm{c}) .
\end{aligned}
$$

Here the time scales are given as $\tau_{\beta, J}=\left(G_{\beta} k_{\beta}\right)^{(-1 / \beta)}$ and $\tau_{\beta}=$ $\left(G_{\infty, \beta} k_{\beta}\right)^{(-1 / \beta)}$ in terms of the quantities defining the individual model components.

When constructing a combined model for the alpha and beta mechanical relaxation, it is natural to try with an additive model in the measured quantity, which in this case is the shear modulus. The two possible additive models consisting of the generalized BEL model and one of the Cole-Cole type models from Fig. 3 are shown in Fig. 4a and b.

Model (a) of Fig. 4 has an obvious problem, namely that the modulus does not decrease to zero in the low-frequency limit because the two springs of the beta part will always be seen; likewise the model does not allow for a dc flow. Model (b) also has problems. In this case the stress does relax to zero at long times, but there is no finite $\mathrm{dc}$ viscosity because of the constant-phase element. The extreme case $\beta=1$ is an exception, but this corresponds to a beta 
a)

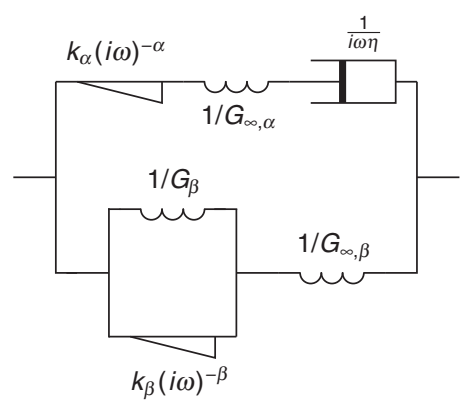

b)

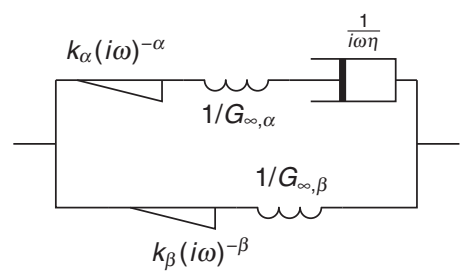

c)

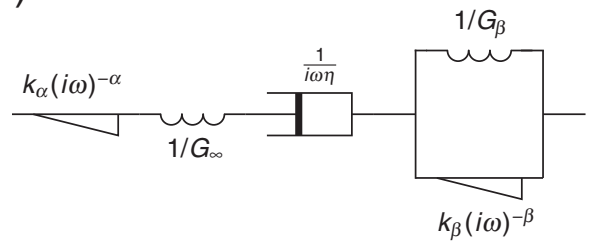

Fig. 4. Mechanical network representation of the models for the full alpha and beta relaxation. The shear compliance is indicated for the individual elements. a) and b) mechanical models which are additive in the shear modulus. c) mechanical model which is additive in the shear compliance (modulus expressed in Eq. (7)).

relaxation with a Debye shape, which is not observed. In conclusion, neither of the two additive models comply with the physical constraints formulated in Eqs. (1)-(3).

Generally, if additivity is assumed in the shear modulus, the beta element must allow for viscous flow in the low-frequency limit. This unavoidably leads to Debye behavior at low frequencies, in contradiction to what is observed. We therefore conclude that there are no realistic models based on addition of the shear modulus of an alpha and beta element.

The alternative is to use a model that is additive in the shear compliance [43], i.e., is a mechanical series combination of the basic elements. Fig. 4c shows such a combination of the generalized BEL model and model (b) from Fig. 3 (note that the two springs in direct series have been merged into one, $1 / G_{\infty}=1 / G_{\infty, \alpha}+1 / G_{\infty, \beta}$ ). This model has physically realistic properties, as it has an elastic behavior at high frequencies and a viscous flow at low frequencies. ${ }^{3}$

The full expression for the shear modulus of our model, which obeys the fundamental requirements, (Fig. 4c) is given by

$$
G(\omega)=\frac{G_{\infty}}{1+\frac{1}{i \omega \tau_{\alpha}}+q\left(\frac{1}{i \omega \tau_{\alpha}}\right)^{\alpha}+\frac{G_{\infty} / G_{\beta}}{1+\left(i \omega \tau_{\beta, J}\right)^{\beta}}} .
$$

\footnotetext{
${ }^{3}$ An model alternative, to the one discussed in detail, consists of the generalized BEL model in series with model (c) from Fig. 3. This does, however, not lead to a model function which resembles the alpha-beta merging phenomenology. The reason is that the model effectively has only one parameter determining both time scale and strength of the beta relaxation part.
}

In the limit $\tau_{\alpha} \rightarrow \infty$ we regain the original beta model (with $G_{\infty, \beta}=G_{\infty}$ ). If $\tau_{\beta, J} \rightarrow \infty$, we likewise recover the original alpha model (with $G_{\infty, \alpha}=G_{\infty}$ ).

The phenomenology of this model is quite different from that of a model that is additive in the shear moduli. The most significant difference is that the beta relaxation does not show up on the lowfrequency side of the alpha relaxation if the beta process happens to take place at lower frequencies than the alpha process. In the work of Sağlanmak et al. [35] a dielectric model based on this shearmechanical model is discussed in detail, ${ }^{4}$ and most of the features can be directly transfered to this pure shear-mechanical model.

Models capturing the alpha-beta merging are often used to investigate the time scale and strength of the beta relaxation corrected for the influence of the alpha relaxation. For such models it is often possible to analytically determine the loss-peak frequency in both the shear modulus and shear compliance of the pure beta model. For our model (the $\tau_{\alpha} \rightarrow \infty$ limit of Eq. (7), corresponding to Eq. (5) with the modified high-frequency spring) it is found that

$\omega_{\mathrm{lp}, \beta, J}=\frac{1}{\tau_{\beta, J}}$

$\omega_{\mathrm{lp}, \beta, G}=\left(\frac{G_{\infty}+G_{\beta}}{G_{\beta}}\right)^{\frac{1}{\beta}} \frac{1}{\tau_{\beta, J}}$.

The difference between the two time scales illustrates that the observed time scale depends significantly on which response function is probed. From the pure beta model it is furthermore possible to find the low-frequency limit of the shear modulus as

$G_{\beta, 0}=\frac{G_{\beta} G_{\infty}}{G_{\beta}+G_{\infty}}$,

and hence the apparent beta relaxation strength $\Delta G_{\beta}=G_{\infty}-G_{\beta, 0}$.

\section{Fitting the new model for the combined shear-mechanical alpha-beta spectrum to data}

To test the ability to fit data with the combined model as expressed in Eq. (7) and illustrated in Fig. 4c, the model was fitted to the ten lowest temperatures of the Polyisobutylene (PIB680) data (Fig. 1). This illustrates what can be learned from fitting a physically reasonable merging model to shear-mechanical data.

The model has seven parameters, four related to the model for the pure alpha relaxation and three to the beta model. This may seem as a large number. However, it should be noted that an alpha model, which has independent control over the high-frequency slope and the width of the peak (or two other independent shape parameters, as in the Havriliak-Negami equation), needs at least four parameters. Likewise, a Cole-Cole type model needs three parameters determining position, strength and shape.

The model can easily be fitted to spectra with well separated alpha and beta relaxations (the lowest temperatures). When the alpha and beta relaxations are close to merging, however, the limited frequency range of the available shear-mechanical data makes this impossible.

Some of us have proposed that the generic alpha relaxation process obeys time-temperature superposition (TTS) and has a high-frequency slope of $-1 / 2[18,44]$. This was originally based on dielectric data, but we later showed it to hold reasonably well for shear-mechanical data as well $[8,11]$. The beta process is furthermore often assumed to have a temperature-independent shape, as discussed above (see also Ref. [25]).

\footnotetext{
${ }^{4}$ The network model for dielectric relaxation discussed in Ref. [35] may be regarded as a combination of this shear-mechanical model with the Gemant-DiMarzio-Bishop model. For a detailed analysis of the validity of the Gemant-DiMarzio-Bishop model see Ref. [29].
} 
Based on these observations the following fitting scheme was applied. At the lowest temperature a least-squares fit was performed with all parameters free except $\alpha=0.5$. Fig. 5 shows the result of this fit. The remaining temperatures were then fitted with the shapedetermining parameters, $q$ and $\beta$, fixed to the found values. Fig. 5 shows also the result of these fits at a higher temperature.

Fig. 6 shows $\tau_{\alpha}, \tau_{\beta, \text { J }}$, and the time scale of the pure shear beta relaxation expressed as $\tau_{\beta, G}=1 / \omega_{\mathrm{lp}, \beta, G}$ (see Eq. (9)). The shear compliance time scale, $\tau_{\beta, \text {, }}$, is almost temperature independent, while the shear modulus time scale, $\tau_{\beta, G}$, is rather temperature dependent. The elastic parameters of the instantaneous modulus, $G_{\infty}$, and the beta spring, $G_{\beta}$, are also reported in Fig. 6 , together with the apparent beta relaxation strength.

The results shown here illustrate that hypothesis regarding spectral shape and the temperature dependence can be tested with the use of such an alpha-beta merging model. The present example shows that within the framework of this merging model, data on Polyisobutylene (PIB680) are consistent with the assumed high-frequency slope of the alpha peak of $-1 / 2$ and a temperatureindependent shape of both the alpha and the beta processes.

\section{Conclusions}

The shear-mechanical beta-relaxation phenomenology was investigated based on data for eighteen liquids. The beta relaxation observed in dielectric spectroscopy corresponds to that of shear-
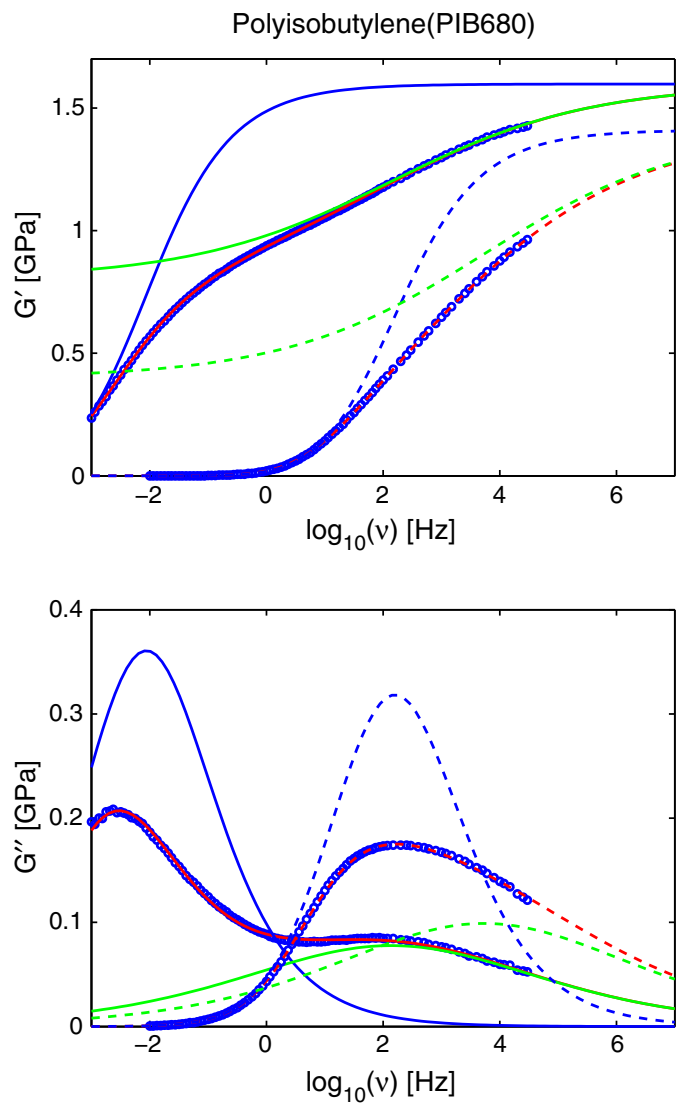

Fig. 5. Fit of the full alpha-beta model function, Eq. (7), to data on Polyisobutylene (PIB680) at two temperatures, $\mathrm{T}=198 \mathrm{~K}$ (full lines) and $216 \mathrm{~K}$ (dashed lines); open circles are raw data. The high-frequency slope of the alpha relaxation was fixed to $-1 / 2$; the additional shape parameters of the alpha and beta relaxation are determined from the fit to the lowest temperature and then fixed for the fit at higher temperature (see section 4 for details on the procedure). Red (gray) lines are the fit of the full alpha-beta model Eq. (7), blue (dark gray) lines the corresponding pure alpha part as given in Eq. (4) with $G_{\infty, \alpha}=G_{\infty}$, green (light gray) lines are the corresponding pure beta part as given in Eq. (5) with $G_{\infty, \beta}=G_{\infty}$.

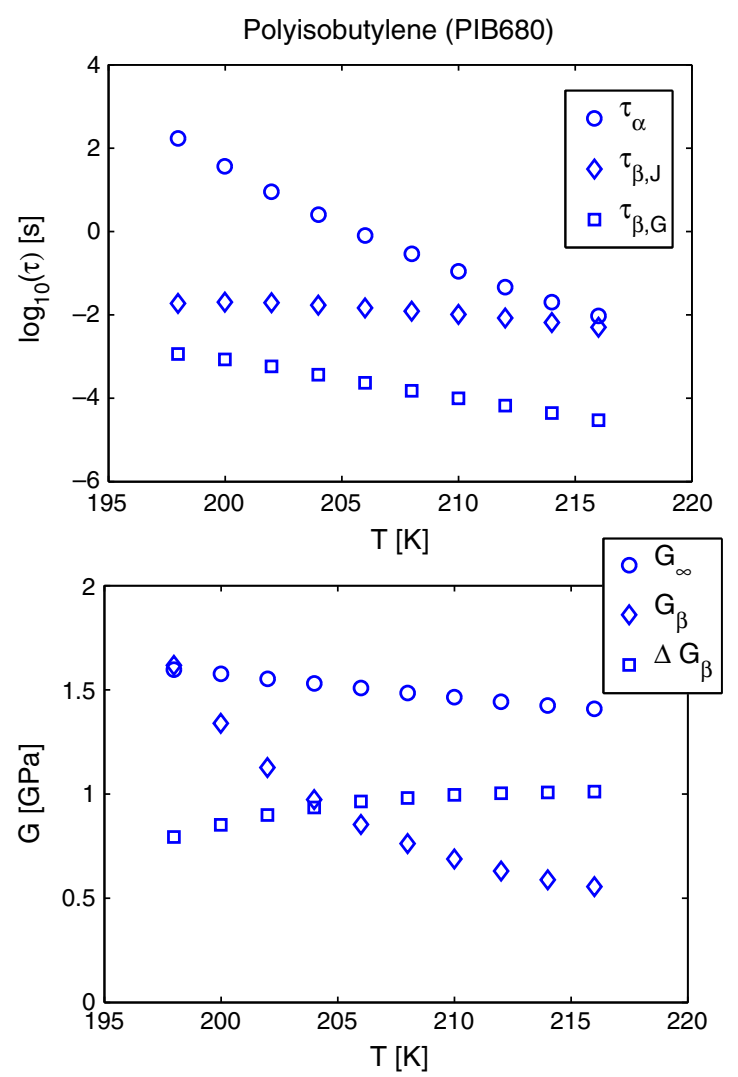

Fig. 6. Resulting parameters from fitting the full alpha-beta model function, Eq. (7), to the shear-mechanical data on Polyisobutylene (PIB680) at the lowest ten temperatures (see section 4 for details on the fitting procedure). Top) The characteristic alpha time scale, $\tau_{\alpha}$, and the two characteristic beta time scales, $\tau_{\beta, J}$ and $\tau_{\beta, G}$. Bottom) Highfrequency elastic modulus, $G_{\infty}$, modulus of beta model spring, $G_{\beta}$, and the apparent beta relaxation strength $\Delta G_{\beta}=G_{\infty}-G_{\beta, 0}$ (with $G_{\beta, 0}$ given in Eq. (10)).

mechanical spectroscopy and vice versa. Just as for the alpha process, there is also a separation of the observed characteristic time scales of the beta process in shear mechanics and dielectrics, with the shear response being somewhat faster in both the alpha and beta cases. The relaxation strength of the beta process relative to the alpha relaxation strength is larger for shear than for dielectric relaxation.

Within the class of viscoelastic models constructed from basic elements a physically sound model must be additive in the shear compliances, not in the shear moduli. A specific model was constructed consisting of a generalized BEL model for the alpha relaxation and a Cole-Cole type model for the beta. This model was fitted to data on Polyisobutylene (PIB680); it was shown that good fits can be obtained under the assumptions of a high-frequency slope of $-1 / 2$ of the alpha relaxation and temperature independent shape parameters for both the alpha and the beta relaxations. This substantiates the conjecture that a $-1 / 2$ slope of the alpha loss (in a log-log plot), as well as TTS, are generic features of the alpha process, and that deviations from this derives from interference of the beta process $[18,44]$.

\section{Acknowledgments}

The centre for viscous liquid dynamics "Glass and Time" is sponsored by the Danish National Research Foundation (DNRF).

\section{References}

[1] B. Jakobsen, C. Maggi, T. Christensen, J.C. Dyre, Investigation of the shearmechanical and dielectric relaxation processes in two monoalcohols close to the glass transition, J. Chem. Phys. 129 (2008) 184502. 
[2] R. Richert, K. Duvvuri, L.T. Duong, Dynamics of glass-forming liquids. VII. Dielectric relaxation of supercooled tris-naphthylbenzene, squalane, and decahydroisoquinoline, J. Chem. Phys. 118 (2003) 1828.

[3] T. Christensen, N.B. Olsen, A rheometer for the measurement of a high-shear modulus covering more than 7 decades of frequency below $50 \mathrm{khz}$, Rev. Sci. Instrum. 66 (1995) 5019.

[4] B. Igarashi, T. Christensen, E.H. Larsen, N.B. Olsen, I.H. Pedersen, T. Rasmussen, J.C. Dyre, A cryostat and temperature control system optimized for measuring relaxations of glass-forming liquids, Rev. Sci. Instrum. 79 (2008) 045105

[5] B. Igarashi, T. Christensen, E.H. Larsen, N.B. Olsen, I.H. Pedersen, T. Rasmussen, J.C. Dyre, An impedance-measurement setup optimized for measuring relaxations of glass-forming liquids, Rev. Sci. Instrum. 79 (2008) 045106

[6] T. Christensen, N.B. Olsen, Comparative measurements of the electrical and shear mechanical response function in some supercooled liquids, J. Non-Cryst. Solids 172-174 (1994) 357.

[7] T. Christensen, N.B. Olsen, Quasistatic measurement of the frequenct-dependent bulk and shear modulus of supercooled liquids, J. Non-Cryst. Solids 172-174 (1994) 362.

[8] B. Jakobsen, K. Niss, N.B. Olsen, Dielectric and shear mechanical alpha and beta relaxations in seven glass-forming liquids, J. Chem. Phys. 123 (2005) 234511.

[9] "Glass and Time: Data repository", Found online at: http://glass.ruc.dk/data

[10] C.F. Behrens, T.G. Christiansen, T. Christensen, J.C. Dyre, N.B. Olsen, Dynamic viscosity of a simple glass-forming liquid - comment, Phys. Rev. Lett. 76 (1996) 1553

[11] C. Maggi, B. Jakobsen, T. Christensen, N.B. Olsen, J.C. Dyre, Supercooled liquid dynamics studied via shear-mechanical spectroscopy, J. Phys. Chem. B 112 (2008) 16320.

[12] K. Niss, Fast and slow dynamics of glass-forming liquids, Ph.D. thesis, De L'Université de Paris XI, 2007.

[13] A. Mandanici, R. Richert, M. Cutroni, X. Shi, S.A. Hutcheson, G.B. McKenna Relaxational features of supercooled and glassy m-toluidine, J. Non-Cryst. Solids 352 (2006) 4729.

[14] S.A. Hutcheson, G.B. McKenna, The measurement of mechanical properties of glycerol, m-toluidine, and sucrose benzoate under consideration of corrected rheometer compliance: An in-depth study and review, J. Chem. Phys. 129 (2008) 074502.

[15] A. Mandanici, M. Cutroni, Multiple mechanical relaxations in ethylcyclohexane above the glass transition temperature, J. Phys. Chem. B 111 (2007) 10999.

[16] A. Mandanici, M. Cutroni, A trace of the Johari-Goldstein relaxation in the mechanical response of supercooled ethylcyclohexane? Mater. Sci. Eng., A 279 (2009) 521-522.

[17] A. Mandanici, W. Huang, M. Cutroni, R. Richert, Dynamics of glass-forming liquids. xii. dielectric study of primary and secondary relaxations in ethylcyclohexane, J. Chem. Phys. 128 (2008) 124505

[18] A.I. Nielsen, T. Christensen, B. Jakobsen, K. Niss, N.B. Olsen, R. Richert, J.C. Dyre Prevalence of approximate $\sqrt{t}$ relaxation for the dielectric $\alpha$ process in viscous organic liquids, J. Chem. Phys. 130 (2009) 154508.

[19] R. Díaz-Calleja, E. Riande, J.S. Román, Román, Interconversion between mechanical and dielectric relaxations for poly(cyclohexyl acrylate), J. Polym. Sci., Part B: Polym. Phys. 31 (1993) 711.

[20] N. Menon, S.R. Nagel, D.C. Venerus, Dynamic viscosity of a simple glass-forming liquid, Phys. Rev. Lett. 73 (1994) 963.

[21] E. Donth, M. Beiner, S. Reissig, J. Korus, F. Garwe, S. Vieweg, S. Kahle, E. Hempel, K. Schröter, Fine structure of the main transition in amorphous polymers:
Entanglement spacing and characteristic length of the glass transition. Discussion of examples, Macromolecules 29 (1996) 6589.

[22] R.D. Deegan, R.L. Leheny, N. Menon, S.R. Nagel, D.C. Venerus, Dynamic shear modulus of tricresyl phosphate and squalane, J. Phys. Chem. B 1033 (1999) 4066.

[23] K. Schröter, E. Donth, Viscosity and shear response at the dynamic glass transition of glycerol, J. Chem. Phys. 113 (2000) 9101.

[24] K. Schröter, E. Donth, Comparison of shear response with other properties at the dynamic glass transition of different glassformers, J. Non-Cryst. Solids 307-310 (2002) 270

[25] N.B. Olsen, Scaling of beta-relaxation in the equilibrium liquid state of sorbitol, J. Non-Cryst. Solids 235 (1998) 399.

[26] N.B. Olsen, T. Christensen, J.C. Dyre, Beta relaxation of nonpolymeric liquids close to the glass transition, Phys. Rev. E 62 (2000) 4435.

[27] J.C. Dyre, N.B. Olsen, Minimal model for beta relaxation in viscous liquids, Phys. Rev. Lett. 91 (2003) 155703

[28] P. Debye, Polar Liquids, The Chemical Catalog Company, Inc, 1929.

[29] K. Niss, B. Jakobsen, N.B. Olsen, Dielectric and shear mechanical relaxations in glass-forming liquids: A test of the Gemant-DiMarzio-Bishop model, J. Chem. Phys. 123 (2005) 234510

[30] A. Gemant, The conception of a complex viscosity and its application to dielectrics, Trans. Faraday Soc. 31 (1935) 1582.

[31] E.A. DiMarzio, M. Bishop, Connection between macroscopic electric and mechanical susceptibilities, J. Chem. Phys. 60 (1974) 3802.

[32] J.C. Dyre, Some remarks on ac conduction in disordered solids, J. Non-Cryst. Solids 135 (1991) 219.

[33] K.L. Ngai, R.W. Rendell, Interpreting the real part of the dielectric permittivity contributed by mobile ions in ionically conducting materials, Phys. Rev. B 61 (2000) 9393.

[34] D.L. Sidebottom, B. Roling, K. Funke, Ionic conduction in solids: Comparing conductivity and modulus representations with regard to scaling properties, Phys. Rev. B 63 (2000) 024301.

[35] N. Sağlanmak, A.I. Nielsen, N.B. Olsen, J.C. Dyre, K. Niss, An electrical circuit model of the alpha-beta merging seen in dielectric relaxation of ultraviscous liquids, J. Chem. Phys. 132 (2010) 024503.

[36] G. Harrison, The Dynamic Properties of Supercooled Liquids, Academic press (INC, London LTD, 1976

[37] K.S. Cole, R.H. Cole, Dispersion and absorption in dielectrics i. alternating current characteristics, J. Chem. Phys. 9 (1941) 341.

[38] A.K. Jonscher, Hopping losses in polarizable dielectric media, Nature 250 (1974) 191.

[39] A.K. Jonscher, The universal dielectric response, Nature 267 (1977) 673.

[40] A.K. Jonscher, Dielectric Relaxation in Solids, Chelsea Dielectric Press Ltd, London, 1983.

[41] A.J. Barlow, A. Erginsav, J. Lamb, Viscoelastic relaxation of supercooled liquids. ii, Proc. R. Soc. London, Ser. A, Mat. Phys, Sciences 298 (1967) 481.

[42] R. Böhmer, G. Diezemann, B. Geil, G. Hinze, A. Nowaczyk, M. Winterlich, Correlation of primary and secondary relaxations in a supercooled liquid, Phys. Rev. Lett. 97 (2006) 135701.

[43] J.D. Ferry, Viscoelastic properties of polymers, John Wiley, 1970.

[44] N.B. Olsen, T. Christensen, J.C. Dyre, Time-temperature superposition in viscous liquids, Phys. Rev. Lett. 86 (2001) 1271. 